\title{
Commentary
}

\section{Endometrial Cells Get Side-Tracked}

\section{Side Population Cells Promote Epithelial-Mesenchymal Transition in Endometrial Carcinoma}

\author{
Martin Götte \\ From the Department of Gynecology and Obstetrics, Münster \\ University Hospital, Münster, Germany
}

Like stem cells, a subpopulation of cancer cells is characterized by the property of self-renewal, unlimited and high proliferative potential, expression of multidrug-resistance proteins, active DNA repair capacity, apoptosis resistance, and an enormous developmental plasticity. ${ }^{1}$ Based on these observations, the concept of cancer stem cells has been developed, which proposes that tumor-initiating cancer cells may either originate from normal stem cells, or that cancer cells may undergo progressive de-differentiation, ultimately leading to a stem-cell like state. The stem cell-like properties of tumorinitiating cells are thought to be a major underlying cause of therapeutic resistance and tumor relapse; therefore, a specific targeting of cancer stem cells within a mixed tumor cell population may represent a particularly efficient form of anticancer therapy.

In this issue of The American Journal of Pathology, Kato et $\mathrm{al}^{2}$ have exploited a common property of stem cells and tumor-initiating cells, the side-population phenotype, to isolate and functionally characterize a cancer stem cell-like subpopulation of endometrial carcinoma. Side population (SP) cells can be identified by flow cytometry based on their property of effluxing the fluorescent dye Hoechst 33342 via ATP-binding cassette transporter proteins such as $A B C G / B r c p 1 .{ }^{3}$ Stem cells express high levels of this protein, and are therefore highly enriched in the SP of a given tissue. High expression of ABCG/Brcp1 by cancer stem cells is thought to be an underlying cause of resistance to chemotherapy, as this protein allows for a rapid clearance of therapeutic drugs from the cells cytoplasm. ${ }^{1,3}$

When Kato et $\mathrm{al}^{2}$ used flow cytometry on primary endometrial cancer cells and the established endometrial cancer cell line Hec1, they were able to identify a small percentage $(<1 \%)$ of SP cells. Isolation and further cultivation of Hec1-derived SP and non-SP cells demon- strated that the SP cells were capable of asymmetric cell division, generating both SP and non-SP cells, which is one hallmark of stem cells. In contrast, the non-SP cells could only generate non-SP cells, but no SP cells, in accordance with a limited differentiation potential. Further analysis revealed that the SP cells expressed lower levels of the differentiation markers CD9 and CD13 compared with non-SP cells. Of note, SP cells showed a high proliferative capacity, and they were capable of dividing for at least 2 months, whereas non-SP cells stopped growing after 2 weeks of culture in mesenchymal stem cell maintenance medium. Similar results were obtained when SP and non-SP cells from an activated $\left({ }^{12} \mathrm{Val}\right) \mathrm{K}$-Ras-transformed rat endometrial cell line were analyzed. Plating of Hec1 SP and non-SP cell lines at a low, clonal density resulted in colony formation in the case of SP cells only. These cells also showed self-renewal properties as they could be serially cloned with similar cloning efficiencies, ${ }^{2}$ which is an additional hallmark of stem cell function. ${ }^{4}$

The isolation of a subpopulation of endometrial cancer cells with stem cell-like properties by Kato et $\mathrm{al}^{2}$ is in accordance with previous reports on stem cell-like properties of endometrial carcinoma cells. For example, the Wnt- $\beta$-catenin and PTEN signal transduction pathways, which are important contributors to adult stem cell selfrenewal and maintenance of "stemness," are frequently dysregulated in endometrial carcinoma. ${ }^{5,6}$ Moreover, increased expression and activity of telomerase, an important factor in conferring unlimited proliferative potential to stem cells, has been observed in endometrial carcinoma. ${ }^{7}$ The finding of an increased proliferative potential in the endometrial cancer cell-derived SP is also in accordance with recent reports of significantly increased numbers of cells expressing the adult stem cell marker Musashi-1 in endometrial carcinoma and proliferative phase endometrium compared with secretory phase en-

Accepted for publication August 19, 2009.

Address reprint requests to Martin Götte, Ph.D., Department of Gynecology and Obstetrics, Münster University Hospital, Albert-Schweitzer-Str. 33, D-48149 Münster, Germany. E-mail: martingotte@uni-muenster.de. 
dometrium. ${ }^{8}$ In contrast, the authors of a second study on endometrial carcinoma SP cells have recently reported the presence of SP cells with a lower proliferation rate compared with non-SP cells. ${ }^{9}$ While this seemingly controversial finding could be explained by methodological differences and the use of different endometrial carcinoma cell lines, it does not necessarily constitute a contradiction, since different hierarchies of stem cells are known to exist. For example, slow cycling stem cells with a pluripotent developmental potential can give rise to highly proliferative transient amplifying cells with more restricted, yet still multipotent developmental capacity. ${ }^{10}$

To test the pathophysiological relevance of their findings in vivo, Kato et $\mathrm{al}^{2}$ monitored growth of Hec1-derived SP and non-SP cells in a nude mice xenograft model. Palpable SP-derived tumors appeared significantly earlier than non-SP tumors and grew significantly faster. Histologically, the SP tumors were more invasive, whereas the non-SP tumors were encapsulated and clearly separated from the basement membrane of the skin. Similar results were obtained when SP and non-SP cells from the activated $\left({ }^{12} \mathrm{Val}\right) \mathrm{K}$-Ras-transformed rat endometrial cell line were used in the in vivo model. Increased invasiveness of the SP cells and increased proliferative potential could also be confirmed in vitro in time-lapse microscopy studies, since SP cells showed lamellipodia formation at the leading edge, and uropodia formation at the trailing edge, which was accompanied by prominent cell migration. In contrast, non-SP cells showed neither podia formation, nor prominent migration.

The observation that the SP-derived tumors were surrounded by stromal tissues with a highly enriched extracellular matrix in both in vivo models prompted Kato et al ${ }^{2}$ to address the question if a fraction of the SP cells may have undergone epithelial to mesenchymal transition (EMT), based on the concept of a high developmental plasticity of stem cells. Indeed, the authors were able to demonstrate increased expression of the mesenchymal cell markers vimentin and $\alpha$-smooth muscle actin ( $\alpha$-SMA), as well as the extracellular matrix protein collagen III in $\mathrm{SP}$ cell tumors compared with non-SP cell derived tumors. Of note, DNA sequencing of microdissected CD9 ${ }^{+}$ tumor and $\alpha$-SMA $/ \mathrm{CD} 13^{+} / \mathrm{CD}^{-}$stroma cell DNA revealed the presence of human $\mathrm{K}$-ras gene sequences in the murine host tissue, demonstrating the SP cell origin of both tumor and stroma cells. These data were complemented by a fluorescence in situ hybridization analysis, which also demonstrated the presence of a high percentage of SP-derived mesenchymal cells in the tumor stroma. Finally, the authors were able to differentiate endometrial carcinoma SP cells into cells of the smooth muscle cell lineage in vitro, whereas this approach was not successful in the case of non-SP cells. ${ }^{2}$ Taken together, the authors' findings suggest that SP cells within an endometrial carcinoma give rise to a highly proliferative and invasive cancer cell population, to tumor stroma cells, and to the secreted matrix surrounding the tumor.

The authors' findings have several important implications (Figure 1). Most notably, the data suggest that SP-derived endometrial cancer stem cells may be able to generate their own stroma, including not only the extracellular matrix, but also stromal cells (Figure 1A). This is a highly relevant finding, which sheds new light on studies focusing on tumor-stroma interactions: If endometrial cancer stem cells are capable of de novo synthesis of their own stem cell niche, they may be far more independent from host tissue composition and properties than previously thought. Future studies need to address if and how the tumor stroma of SP-derived tumors differs from the stroma of non-SP derived tumors. The identification of increased collagen III biosynthesis in SP-derived tumors by Kato et $\mathrm{al}^{2}$ is a first step in this direction. Other com-

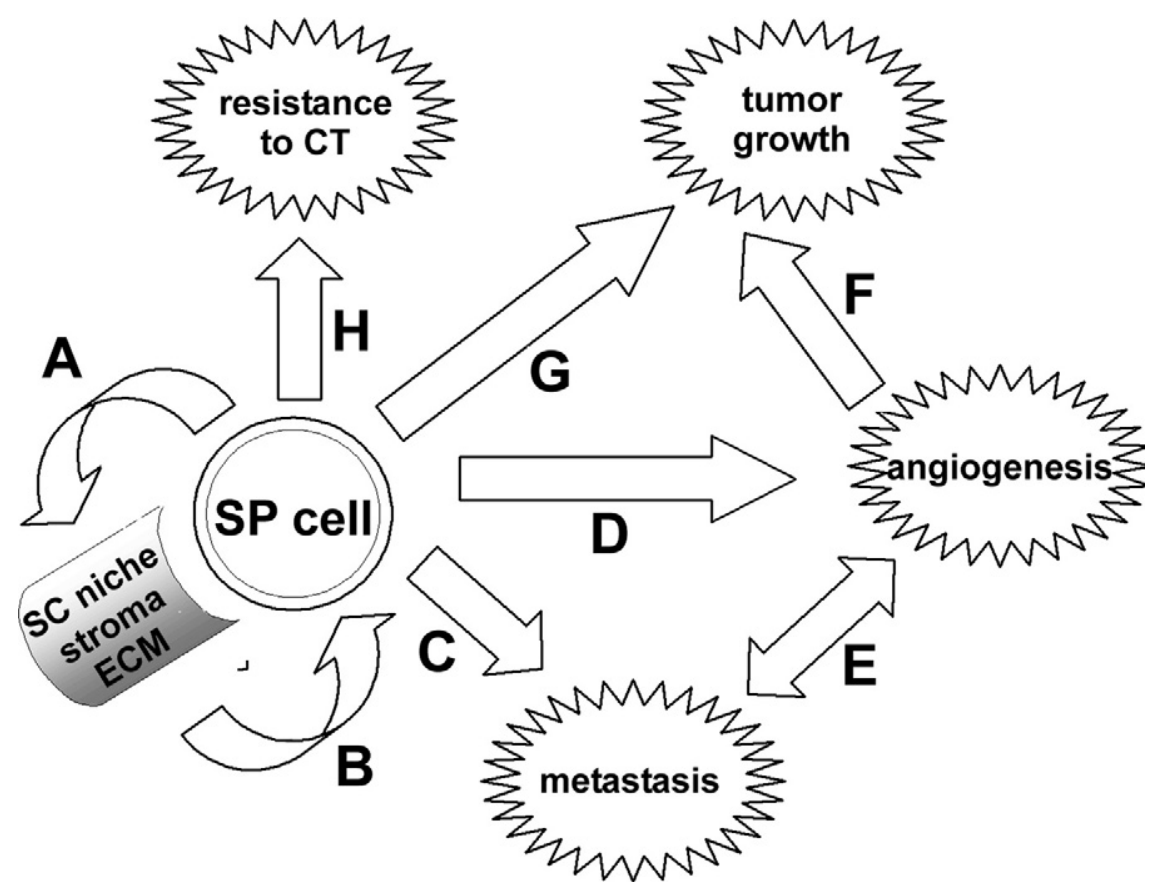

Figure 1. Contribution of SP cells to endometrial carcinoma pathogenesis. A: EMT, promoted by the high developmental plasticity of stem cells (SC), results in formation of an extracellular matrix (ECM)-rich tumor stroma. B: The tumor stroma serves as a stem cell niche, which is required to maintain the undifferentiated character of the cancer stem cells. C: Through EMT, SP cells loose contact inhibition and acquire a highly migratory phenotype, which facilitates metastasis. D: EMT may promote differentiation to mesenchymal endothelial or mural cells, promoting tumor angiogenesis. E: Angiogenesis will facilitate tumor cell dissemination into the blood stream, thus promoting metastasis. F: Angiogenesis is a prerequisite for primary tumor growth G: The highly proliferative nature and the unlimited potential for cell division of SP cells displaying a transient amplifying cell phenotype promotes tumor growth. H: The expression of multidrug resistance proteins increases SP cell resistance to chemotherapy (CT). 
ponents of the extracellular matrix, such as proteoglycans, may also be of relevance as they play major supportive roles in developmental signaling and provide a niche for preservation of the undifferentiated state of stem cells. ${ }^{11}$ For example, mice deficient in the cell surface proteoglycan syndecan-1 (CD138), a molecular marker associated with epithelial-mesenchymal transition during development and carcinogenesis, ${ }^{12}$ were shown to be resistant to a variety of experimentally induced cancers due to a reduction in a wnt-responsive progenitor cell population. ${ }^{11,13}$ In human endometrial carcinoma, low epithelial CD138 expression is correlated with negative prognostic factors and disease stage, whereas stromal CD138 expression was shown to be significantly higher in high-grade tumors and to be an independent prognostic factor for both disease-free and overall survival. ${ }^{14}$ Future studies need to address to which extent the stem cellrelated functions of CD138 determine its role in endometrial cancer.

While the study by Kato et $\mathrm{al}^{2}$ indicates that the SPderived endometrial cancer cells may autonomously establish their own stem cell niche, the niche itself feeds back to the stem cell and keeps it in an undifferentiated state $^{15}$ (Figure 1B). An additional implication of the high developmental plasticity of endometrial carcinoma SP cells is a facilitation of EMT, which induced a highly mobile, migratory phenotype. ${ }^{2}$ While not experimentally tested in the study by Kato et al, ${ }^{2}$ it can be expected that this phenotype will promote metastasis of endometrial carcinoma cells (Figure 1C). Similarly, EMT may promote angiogenesis if SP cells differentiate into endothelial or mural cells, as indicated by the induced transformation of endometrial cancer-derived SP cells into $\alpha$-SMA-positive cells (Figure 1D). ${ }^{2}$ Angiogenesis would in turn further promote dissemination of tumor cells (Figure 1E) and would be a requirement for sustained primary tumor growth (Figure 1F). This is reflected by the authors' observation of a higher vascularization of SP-derived tumors as compared with non-SP tumors based on $\alpha$-SMA staining. Apart from indirect, angiogenesis-related effects, SP cells within endometrial carcinomas displayed a particularly aggressive phenotype with a high proliferative potential reminiscent of transient amplifying cells, ${ }^{10}$ which directly promoted primary tumor growth ${ }^{2}$ (Figure 1G). Finally, while not directly addressed in the study by Kato et al, ${ }^{2}$ by definition of the SP phenotype, the SP subpopulation of endometrial carcinoma cells can be expected to display higher resistance to chemotherapy, due to the expression of multidrug resistance transporter proteins such as $A B C G / B r c p 1^{1,3}$ (Figure $1 \mathrm{H}$ ). In fact, a recent study on SP and non-SP subpopulations of the endometrial carcinoma cell line AN3CA revealed increased resistance of the SP cells to paclitaxel, supporting this hypothesis. ${ }^{9}$

From a therapeutic perspective, the study by Kato et $\mathrm{al}^{2}$ has additional implications. As SP cells modulate a multitude of processes relevant to endometrial carcinoma pathogenesis, including persistent primary tumor cell proliferation, promotion of tumor angiogenesis and metastasis, and resistance to chemotherapy, a selective targeting of cancer stem cells should hold a lot of prom- ise, simultaneously blocking a number of relevant pathogenetic processes. A more conventional approach could lie in the identification of cancer stem cells by antibodyor small molecule-based in vivo imaging techniques, followed by radiotherapy. However, it was proposed that cancer stem cells may be more resistant to radiotherapy, ${ }^{16}$ which is a property that may hamper rapid implementation of this concept. An alternative approach is the forced differentiation of cancer stem cells, which is aimed at inducing a loss of stem-cell like properties in the target cells, followed by conventional therapeutic intervention. ${ }^{1,11}$ This approach has yielded very promising results in experimental cancer models, ${ }^{17,18}$ however, translation into clinical applications has not yet been achieved.

Apart from technical problems of implementation, a major caveat is associated with stem-cell targeting therapies of the endometrium, particularly in premenopausal women: during reproductive life, the human endometrium is a highly dynamic tissue that constantly undergoes cycles of growth, differentiation, shedding, and regeneration. In fact, increasing evidence suggests that stem cells are responsible for the tremendous regenerative capacity of the human endometrium, which is growing from 0.5 to $1 \mathrm{~mm}$ following menstruation to 5 to $7 \mathrm{~mm}$ in thickness each menstrual cycle. ${ }^{4,8,15}$ Consequently, endometrial stem cell activity is required for proper physiological function of the endometrium. A targeting of endometrial cancer stem cells may therefore lead to massive side effects, possibly leading to infertility and endometrial dysfunctions typically not associated with premenopausal women if physiological endometrial stem cells would also be affected by these therapeutic approaches. Therefore, future studies need to define differences between physiological endometrial stem cells and endometrial cancer stem cells, to develop targeted therapies.

The increasing knowledge on the regulatory function of microRNAs, which are posttranscriptional modulators of gene expression, may assist in the development of novel therapies: It was recently shown that a single microRNA, miR-145, synchronously regulates the expression of the pluripotency-associated transcription factors OCT4, SOX2, and KLF4, which are key factors in the generation of induced pluripotent stem cells. ${ }^{19}$ In human embryonic stem cells, increased miR-145 expression inhibited selfrenewal, repressed expression of pluripotency genes, and induced lineage-restricted differentiation. ${ }^{19}$ Of note, both OCT4 ${ }^{20}$ and SOX2 (M Götte, M Wolf, AN Schüring, $\mathrm{L}$ Kiesel, unpublished data) are expressed by putative endometrial stem cells. Moreover, although a dysregulation of miR-145 expression in endometrial cancer has not been reported so far, its expression is up-regulated in endometriosis, a proliferative disease of the endometrium characterized by the growth of ectopic implants outside the uterine cavity, ${ }^{21}$ suggesting that investigation of miR145 function may also be worthwhile in endometrial carcinoma. Future studies need to address if manipulation of microRNA function may be a practicable approach in the synchronous targeting of cancer stem cell functions. This concept may be not too far removed from clinical applications, since intraperitoneal injection of microRNA inhibitors in nonhuman primates has already proven to be an 
efficient and side-effect free experimental therapeutic strategy for lowering high cholesterol levels. ${ }^{22}$

In conclusion, the study by Kato et $\mathrm{al}^{2}$ highlights the relevance of endometrial carcinoma SP cell dysregulation for a multitude of processes associated with endometrial carcinogenesis. A detailed molecular characterization of endometrial cancer stem cells will help to establish novel diagnostic markers, and prognostic values. Furthermore, a selective targeting of cancer cells displaying stem-cell like properties through induced differentiation of cancer stem cells, or through innovative combination of molecular diagnostics with conventional therapies holds a lot of promise. However, future therapeutic approaches need to consider and overcome possible side effects associated with a targeting of endometrial SP cells, since the endometrium is a highly regenerative tissue that depends on physiological stem cell function. ${ }^{4,8,15}$

\section{References}

1. Pardal R, Clarke MF, Morrison SJ: Applying the principles of stem-cell biology to cancer. Nat Rev Cancer 2003, 8:895-902

2. Kato K, Takao T, Kuboyama A, Tanaka A, Ohgami T, Yamaguchi S, Adachi S, Yoneda T, Ueoka Y, Kato K, Hayashi S, Asanoma K, Wake $\mathrm{N}$ : Endometrial cancer side-population cells show prominent migration and have a potential to differentiate into the mesenchymal cell lineage. Am J Pathol 2010, 176:381-392

3. Zhou S, Schuetz JD, Bunting KD, Colapietro AM, Sampath $H$, Sorrentino BP: The ABC transporter Bcrp1/ABCG2 is expressed in a wide variety of stem cells and is a molecular determinant of the sidepopulation phenotype. Nat Med 2001, 7:1028-1034

4. Gargett CE, Schwab KE, Zillwood RM, Nguyen HP, Wu D: Isolation and culture of epithelial progenitors and mesenchymal stem cells from human endometrium. Biol Reprod 2009, 80:1136-1145

5. Moreno-Bueno G, Hardisson D, Sánchez C, Sarrió D, Cassia R, García-Rostán G, Prat J, Guo M, Herman JG, Matías-Guiu X, Esteller $\mathrm{M}$, Palacios $\mathrm{J}$ : Abnormalities of the APC/ $\beta$-catenin pathway in endometrial cancer. Oncogene 2002, 21:7981-7990

6. Zhou C, Bae-Jump VL, Whang YE, Gehrig PA, Boggess JF: The PTEN tumor suppressor inhibits telomerase activity in endometrial cancer cells by decreasing hTERT mRNA levels. Gynecol Oncol 2006, 101:305-310

7. Kyo S, Kanaya T, Takakura M, Tanaka M, Inoue M: Human telomerase reverse transcriptase as a critical determinant of telomerase activity in normal and malignant endometrial tissues. Int J Cancer 1999, 80:60-63

8. Götte M, Wolf M, Staebler A, Buchweitz O, Kelsch R, Schüring AN Kiesel L: Increased expression of the adult stem cell marker Musashi-1 in endometriosis and endometrial carcinoma. J Pathol 2008, 215:317-329

9. Friel AM, Sergent PA, Patnaude C, Szotek PP, Oliva E, Scadden DT, Seiden MV, Foster R, Rueda BR: Functional analyses of the cancer stem cell-like properties of human endometrial tumor initiating cells. Cell Cycle 2008, 7:242-249

10. Miller SJ, Lavker RM, Sun TT: Interpreting epithelial cancer biology in the context of stem cells: tumor properties and therapeutic implications. Biochim Biophys Acta 2005, 1756:25-52

11. Yip GW, Smollich M, Götte M: Therapeutic value of glycosaminoglycans in cancer. Mol Cancer Ther 2006, 5:2139-2148

12. Götte M, Kersting C, Radke I, Kiesel L, Wülfing P: An expression signature of Syndecan-1 (CD138). E-cadherin and c-met is associated with factors of angiogenesis and lymphangiogenesis in ductal breast carcinoma in situ. Breast Cancer Res 2007, 9:R8

13. McDermott SP, Ranheim EA, Leatherberry VS, Khwaja SS, Klos KS, Alexander CM: Juvenile syndecan-1 null mice are protected from carcinogen-induced tumor development. Oncogene 2007, 26:1407-1416

14. Hasengaowa, Kodama J, Kusumoto T, Shinyo Y, Seki N, Hiramatsu Y: Prognostic significance of syndecan-1 expression in human endometrial cancer. Ann Oncol 2005, 16:1109-1115

15. Gargett CE, Chan RW, Schwab KE: Hormone and growth factor signaling in endometrial renewal: role of stem/progenitor cells. Mol Cell Endocrinol 2008, 25:22-29

16. Baumann M, Krause M, Hill R: Exploring the role of cancer stem cells in radioresistance. Nat Rev Cancer 2008, 8:545-554

17. Jain M, Arvanitis C, Chu K, Dewey W, Leonhardt E, Trinh M, Sundberg CD, Bishop JM, Felsher DW: Sustained loss of a neoplastic phenotype by brief inactivation of MYC. Science 2002, 297:102-104

18. Sureban SM, May R, George RJ, Dieckgraefe BK, McLeod HL, Ramalingam S, Bishnupuri KS, Natarajan G, Anant S, Houchen CW: Knockdown of RNA binding protein musashi-1 leads to tumor regression in vivo. Gastroenterology 2008, 134:1448-1458

19. Xu N, Papagiannakopoulos T, Pan G, Thomson JA, Kosik KS: MicroRNA-145 regulates OCT4. SOX2, and KLF4 and represses pluripotency in human embryonic stem cells. Cell 2009, 137:647-658

20. Matthai C, Horvat R, Noe M, Nagele F, Radjabi A, van Trotsenburg M, Huber J, Kolbus A: Oct-4 expression in human endometrium. Mol Hum Reprod 2006, 12:7-10

21. Ohlsson Teague EM, Van der Hoek KH, Van der Hoek MB, Perry N, Wagaarachchi P, Robertson SA, Print CG, Hull LM: MicroRNA-regulated pathways associated with endometriosis. Mol Endocrinol 2009, 23:265-75

22. Elmén J, Lindow M, Schütz S, Lawrence M, Petri A, Obad S, Lindholm M, Hedtärn M, Hansen HF, Berger U, Gullans S, Kearney P, Sarnow $P$, Straarup EM, Kauppinen S: LNA-mediated microRNA silencing in non-human primates. Nature 2008, 452:896-899 